GLASNIK MATEMATIČKI

Vol. 40(60)(2005), $333-337$

\title{
D-CONTINUUM $X$ ADMITS A WHITNEY MAP FOR $C(X)$ IF AND ONLY IF IT IS METRIZABLE
}

\author{
IVAN LONČAR \\ University of Zagreb, Croatia
}

\begin{abstract}
The main purpose of this paper is to prove: a) a Dcontinuum $X$ admits a Whitney map for $C(X)$ if and only if it is metrizable, b) a continuum $X$ admits a Whitney map for $C^{2}(X)$ if and only if it is metrizable.
\end{abstract}

\section{INTRODUCTION}

All spaces in this paper are compact Hausdorff and all mappings are continuous. The weight of a space $X$ is denoted by $w(X)$.

A generalized arc is a Hausdorff continuum with exactly two nonseparating points (end points) $x, y$. Each separable arc is homeomorphic to the closed interval $I=[0,1]$.

We say that a space $X$ is arcwise connected if for every pair $x, y$ of points of $X$ there exists a generalized $\operatorname{arc} L$ with end points $x, y$.

Let $X$ be a space. We define its hyperspaces as the following sets:

$$
\begin{aligned}
2^{X} & =\{F \subseteq X: F \text { is closed and nonempty }\}, \\
C(X) & =\left\{F \in 2^{X}: F \text { is connected }\right\} \\
C^{2}(X) & =C(C(X)), \\
X(n) & =\left\{F \in 2^{X}: F \text { has at most } n \text { points }\right\}, n \in \mathbb{N} .
\end{aligned}
$$

For any finitely many subsets $S_{1}, \ldots, S_{n}$, let

$$
\left\langle S_{1}, \ldots, S_{n}\right\rangle=\left\{F \in 2^{X}: F \subset \bigcup_{i=1}^{n} S_{i} \text {, and } F \cap S_{i} \neq \emptyset \text {, for each } i\right\} .
$$

2000 Mathematics Subject Classification. 54F15, 54C30.

Key words and phrases. D-continuum, Whitney map. 
The topology on $2^{X}$ is the Vietoris topology, i.e., the topology with a base $\left\{<U_{1}, \ldots, U_{n}\right\rangle: U_{i}$ is an open subset of $X$ for each $i$ and each $\left.n<\infty\right\}$, and $C(X), X(n)$ are subspaces of $2^{X}$. Moreover, $X(1)$ is homeomorphic to $X$.

Let $X$ and $Y$ be the spaces and let $f: X \rightarrow Y$ be a mapping. Define $2^{f}: 2^{X} \rightarrow 2^{Y}$ by $2^{f}(F)=f(F)$ for $F \in 2^{X}$. By [8, p. 170, Theorem 5.10] $2^{f}$ is continuous and $\left.2^{f}(C(X)) \subset C(Y), 2^{f}(X(n)) \subset Y\right)$. The restriction $2^{f} \mid C(X)$ is denoted by $C(f)$.

A continuum $X$ is called a $D$-continuum if for every pair $C, D$ of its disjoint non-degenerate subcontinua there exists a subcontinuum $E \subset X$ such that $C \cap E \neq \emptyset \neq D \cap E$ and $(C \cup D) \backslash E \neq \emptyset$.

Lemma 1.1. [6, Lemma 2.3]. If $X$ is an arcwise connected continuum, then $X$ is a D-continuum.

Lemma 1.2. [6, Lemma 2.4]. If $X$ is a locally connected continuum, then $X$ is D-continuum.

Let $\Lambda$ be a subspace of $2^{X}$. By a Whitney map for $\Lambda[9$, p. 24, (0.50)] we will mean any mapping $g: \Lambda \rightarrow[0,+\infty)$ satisfying

a) if $A, B \in \Lambda$ such that $A \subset B$ and $A \neq B$, then $g(A)<g(B)$ and

b) $g(\{x\})=0$ for each $x \in X$ such that $\{x\} \in \Lambda$.

If $X$ is a metric continuum, then there exists a Whitney map for $2^{X}$ and $C(X)$ ([9, pp. 24-26], [3, p. 106]). On the other hand, if $X$ is non-metrizable, then it admits no Whitney map for $2^{X}$ [1]. It is known that there exist nonmetrizable continua which admit and ones which do not admit a Whitney map for $C(X)[1]$.

In the sequel we shall use the following theorem.

Theorem 1.3. [6, Theorem 3.3]. If a D-continuum $X$ admits a Whitney map for $C(X)$, then $C(X) \backslash X(1)$ is metrizable and $w(C(X) \backslash X(1)) \leq \aleph_{0}$.

It is known that if $X$ is a continuum, then $C(X)$ is arcwise connected [7, p. 1209, Theorem]. Hence, using Lemma 1.1 and Theorem 1.3, we obtain the following corollary.

Corollary 1.4. If $X$ is a continuum which admits a Whitney map for the hyperspace $C^{2}(X)$, then $C^{2}(X) \backslash C(X)(1)$ is metrizable and

$$
w\left(C^{2}(X) \backslash C(X)(1)\right) \leq \aleph_{0} .
$$

\section{MAIN THEOREMS}

In this section we shall prove the main theorems of the paper, Theorems 2.2 and 2.6 .

For this purpose we shall use the notion of a network of a topological space. 
A family $\mathcal{N}=\left\{M_{s}: s \in S\right\}$ of a subsets of a topological space $X$ is a network for $X$ if for every point $x \in X$ and any neighbourhood $U$ of $x$ there exists an $s \in S$ such that $x \in M_{s} \subset U[2$, p. 170]. The network weight of a space $X$ is defined as the smallest cardinal number of the form $\operatorname{card}(\mathcal{N})$, where $\mathcal{N}$ is a network for $X$; this cardinal number is denoted by $n w(X)$.

Theorem 2.1. [2, p. 171, Theorem 3.1.19]. For every compact space $X$ we have $n w(X)=w(X)$.

Now we shall prove the main theorem of this paper.

Theorem 2.2. A D-continuum $X$ admits a Whitney map for $C(X)$ if and only if it is metrizable.

Proof. If $X$ is metrizable, then $X$ admits a Whitney map ([3, p. 106], [9, pp. 24-26]). Conversely, suppose that $X$ admits a Whitney map for $C(X)$. By Theorem 1.3 we have that $C(X) \backslash X(1)$ is metrizable and $w(C(X) \backslash X(1)) \leq$ $\aleph_{0}$. This means that there exists a countable base $\mathcal{B}=\left\{B_{i}: i \in \mathbb{N}\right\}$ of $C(X) \backslash X(1)$. For each $B_{i}$ let $C_{i}=\left\{x \in X: x \in B, B \in B_{i}\right\}$, i.e., the union of all continua $B$ contained in $B_{i}$.

Claim 1. The family $\left\{C_{i}: i \in N\right\}$ is a network of $X$. Let $X$ be a point of $X$ and let $U$ be an open subsets of $X$ such that $x \in U$. There exists and open set $V$ such that $x \in V \subset \mathrm{Cl} V \subset U$. Let $K$ be a component of $\mathrm{Cl} V$ containing $x$. By Boundary Bumping Theorem $[10$, p. 73 , Theorem 5.4] $K$ is nondegenerate and, consequently, $K \in C(X) \backslash X(1)$. Now, $\langle U\rangle \cap(C(X) \backslash X(1))$ is a neighbourhood of $K$ in $C(X) \backslash X(1)$. It follows that there exists a $B_{i} \in \mathcal{B}$ such that $K \in B_{i} \subset\langle U\rangle \cap(C(X) \backslash X(1))$. It is clear that $C_{i} \subset U$ and $x \in C_{i}$ since $x \in K$. Hence, the family $\left\{C_{i}: i \in N\right\}$ is a network of $X$.

Claim 2. $n w(X)=\aleph_{0}$. Apply Claim 1 and the fact that $\mathcal{B}$ is countable.

Claim 3. $w(X)=\aleph_{0}$. By Claim 2 we have $n w(X)=\aleph_{0}$. Moreover, by Theorem $2.1 w(X)=\aleph_{0}$.

Claim 4. Finally, $X$ is metrizable.

Since each arcwise connected continuum is a D-continuum (Lemma 1.1) we have the following corollary which generalize Theorem 3.4 of the paper [5, p. 19].

Corollary 2.3. An arcwise connected continuum $X$ admits a Whitney map for $C(X)$ if and only if $X$ is metrizable.

An arboroid is a hereditarily unicoherent arcwise connected continuum. A metrizable arboroid is a dendroid. If $X$ is an arboroid and $x, y \in X$, then there exists a unique $\operatorname{arc}[x, y]$ in $X$ with endpoints $x$ and $y$.

A point $t$ of an arboroid $X$ is said to be a ramification point of $X$ if $t$ is the only common point of some three arcs such that it is the only common point of any two, and an end point of each of them. 
If an arboroid $X$ has only one ramification point $t$, it is called a generalized fan with the top $t$. A metrizable generalized fan is called a fan.

The following corollary is a stronger result than Theorem 4.20 in [4] which states that a generalized fan $X$ admits a Whitney map for $C(X)$ if and only if it is metrizable.

Corollary 2.4. Let $X$ be an arboroid. Then $X$ admits a Whitney map for $C(X)$ if and only if it is metrizable.

Proof. Apply Corollary 2.3.

From Lemma 1.2 it follows that each locally connected continuum is a D-continuum. Thus, we have the following corollary of Theorem 2.2.

Corollary 2.5. A locally connected $X$ continuum admits a Whitney map for $C(X)$ if and only if it is metrizable.

The following theorem shows that the existence of a Whitney map for $C^{2}(X)$ is equivalent to metrizability of $X$.

Theorem 2.6. A continuum $X$ admits a Whitney map for $C^{2}(X)$ if and only if $X$ is metrizable.

Proof. From Corollary 1.4 it follows that if $X$ a continuum which admits a Whitney map for $C^{2}(X)$, then $C^{2}(X) \backslash C(X)(1)$ is metrizable and $w\left(C^{2}(X) \backslash C(X)(1)\right) \leq \aleph_{0}$. By Theorem $2.2 w(C(X))=\aleph_{0}$ since $C(X)$ is arcwise connected. This means that $w(X)=\aleph_{0}$ since $X$ is homeomorhic to $X(1) \subset C(X)$. Hence, $X$ is metrizable.

It is known $[2$, p. 171 , Corollary 3.1.20] that if a compact space $X$ is the countable union of its subspaces $X_{n}, n \in \mathbb{N}$, such that $w\left(X_{n}\right) \leq \aleph_{0}$, then $w(X) \leq \aleph_{0}$. Using this fact and theorems proved in the previous section we obtain the following theorems.

THEOREM 2.7. If a continuum $X$ is the countable union either of its $D$ subcontinua or of its arcwise connected subcontinua, then $X$ admits a Whitney map for $C(X)$ if and only if it is metrizable.

THEOREM 2.8. If a compact space $X$ is the countable union of its subcontinua and admits a Whitney map for $C^{2}(X)$, then $X$ is metrizable.

ACKNOWLEDGEMENTS.

The author is very grateful to the referee for his/her help and valuable suggestions.

\section{REFERENCES}

[1] J. J. Charatonik and W. J. Charatonik, Whitney maps-a non-metric case, Colloq. Math. 83 (2000), 305-307.

[2] R. Engelking, General Topology, PWN, Warszawa, 1977. 
[3] A. Illanes and S.B. Nadler, Jr., Hyperspaces: Fundamentals and Recent advances, Marcel Dekker, New York-Basel, 1999.

[4] I. Lončar, $A$ fan $X$ admits a Whitney map for $C(X)$ iff it is metrizable, Glas. Mat. Ser. III 38 (58) (2003), 395-411.

[5] I. Lončar, Arc-smooth continuum admits $X$ admits a Whitney map for $C(X)$ iff it is metrizable, JP J. Geom. Topol. 4 (2004), 13-21.

[6] I. Lončar, Whitney map for hyperspaces of continua with the property of Kelley, JP J. Geom. Topol. 4 (2004), 147-156.

[7] M. M. McWaters, Arcs, semigroups, and hyperspace, Canad. J. Math. 20 (1968), 12071210.

[8] E. Michael, Topologies on spaces of subsets, Trans. Amer. Math. Soc. 71 (1951), 152182.

[9] S. B. Nadler, Hyperspaces of sets, Marcel Dekker, Inc., New York, 1978.

[10] S. B. Nadler, Continuum theory, Marcel Dekker, Inc., New York, 1992.

I. Lončar

Faculty of Organizations and Informatics Varaždin

University of Zagreb

Croatia

E-mail: ivan.loncar1@vz.htnet.hr, ivan.loncar@foi.hr

Received: 4.10.2004. 